\title{
Enriched damage models for continuum failure analyses
}

\author{
Gilles Pijaudier-Cabot* and René de Borst** \\ *Laboratoire de Génie Civil de Nantes Saint-Nazaire \\ Ecole Centrale de Nantes, BP 92101, 44321 Nantes Cedex 03, France \\ Email: Gilles.Pijaudier-Cabot@ec-nantes.fr \\ **Koiter Institute, Delft University of Technology \\ P.O. Box 5058, 2600 GB Delft, The Netherlands
}

\begin{abstract}
Continuum damage mechanics is a framework for describing the variations of the elastic properties of a material due to microstructural degradations. In this contribution two issues are reviewed: the first one deals with standard damage models, damage induced anisotropy and relations with other existing approaches such as smeared crack models. The second issue is concerned with strain localisation due to softening, which is inherent to continuum damage modelling of quasi-brittle materials. Nonlocal approaches to damage are reviewed. We conclude with possible extensions of these enriched damage models to the case of damage induced anisotropy.

Keywords: Damage, isotropy, anisotropy, strain localisation, nonlocality.
\end{abstract}

\section{INTRODUCTION}

Computational modelling and failure analysis of quasi-brittle materials and structures require adequate constitutive models coupled to robust computational schemes. Some of the issues which received most of the attention in this field over the past two decades are progressive cracking and strain localisation due to the inherent softening of the material. With respect to the first item, continuous damage models have become among the most popular ones. Isotropic and anisotropic formulations were proposed. Whether they derive from smeared crack approaches including the rotating crack models [see e.g. Feenstra, 1993], microplane models [Bazant and Prat, 1988], or pure phenomenological constitutive relations [Mazars 1984, Lemaitre 1992], the basic principle remains the same: the compliance of the material can be seen as an internal variable, in the thermodynamic sense, which is indexed on the state of microcracking of the material.

The issue of strain localisation due to strain softening is a bit more intricate since it reflects on the ability of models to capture the inception of failure and its propagation when solving boundary value problems. It became apparent at the end of the 70's that strain softening causes a loss of well posedness of incremental boundary value problems and, more importantly, that it yields prediction of failure without energy dissipation [Bazant, 1976, Rice 1976]. The remedy to this physically unrealistic feature was found 
in the early 90's, surprisingly inspired by earlier theories of materials with microstructure to situations where the material behaviour is not reversible anymore. Among such models, called enriched models for failure analysis, are the nonlocal damage model [Pijaudier-Cabot and Bazant, 1987], gradient plasticity approaches [de Borst and Muhlhaus, 1992], Cosserat models [de Borst, 1991] and more recently gradient damage models [Peerlings et al., 1996]. Their common feature is the incorporation in the constitutive relations of an internal length which controls the failure process and thus precludes any dissipative process to occur in a region of the solid of zero volume.

It is also interesting to observe that, in fluid mechanics, similar issues were faced with transonic flow and boundary layer problems [Joseph et al. 1985]. The most popular techniques for circumventing those problems were the introduction of a characteristic time in the problem, for instance by considering that the fluid is viscous. A small amount of viscosity (damping) provides a regularisation of the governing equations. Similar solutions exist in solid mechanics. Needleman [1988] and Sluys [1992] demonstrated that material rate dependency is a proper regularisation method, i.e. that it restores well posedness of the BVP at the inception of softening. Although the technique is very attractive, meaning that physical motivations for rate dependency might be easier to provide than for nonlocality of constitutive relations, some inconsistencies remain because the response of a quasi brittle material is slightly more complex compared to simple fluids: rate dependency provides for instance an evolution of the material strength for homogeneously strained specimens at different loading rates. At the same time, it provides also a width of the fracture process zone and eventually controls the fracture energy of the material. The fracture energy and the evolution of the material strength with the loading rate are two characteristics which should be fitted with a single parameter (the viscosity), which is not always in accordance with experimental data.

In this chapter, we will concentrate on rate independent modelling of damage and on two regularisation techniques: the nonlocal and gradient approaches. In the first part, we will review standard damage models, isotropic and anisotropic. The second part will be devoted to the integral and gradient enrichments of such models.

\section{STANDARD DAMAGE MODELS}

Let us consider, for the sake of simplicity, the case of tension dominated mechanical actions. The development of microcracks results in a progressive degradation of the material elastic stiffness. In the reversible (elastic) domain, the stress-strain relation reads:

$$
\sigma_{i j}=C_{i j k l}^{\text {damaged }} \varepsilon_{k l},
$$

where $\sigma_{i j}$ is the stress component, $\boldsymbol{\varepsilon}_{\boldsymbol{k} \boldsymbol{l}}$ is the strain component, and $C_{i j k l}^{\text {dumaged }}$ is the stiffness coefficient of the damaged material. The simplest approach to material damage 
is to assume that the material stiffness (for isotropic materials) remains isotropic. The stress strain relations becomes :

$$
\varepsilon_{i j}=\frac{3}{2 E_{0}(1-d)}\left(\sigma_{i j}-\frac{\sigma_{k k}}{3} \delta_{i j}\right)+\frac{\left(1-2 v_{0}\right)}{2 E_{0}(1-D)}\left[\sigma_{k k} \delta_{i j}-\sigma_{i j}\right],
$$

where $E_{0}$ and $\boldsymbol{v}_{\mathbf{0}}$ are the Young's modulus and Poisson's ratio of the undamaged isotropic material, and $\boldsymbol{\delta}_{i j}$ is the kronecker symbol, $d$ and $D$ are two independent damage variables which vary between 0 and 1 . It should be pointed out that isotropic damage means indeed two damage state variables. The subsequent assumption $d=D$ yields the stress-strain relationship used by Mazars (1984):

or

$$
\begin{aligned}
& \varepsilon_{i j}=\frac{1+v_{0}}{E_{0}(1-d)} \sigma_{i j}-\frac{v_{0}}{E_{0}(1-d)}\left[\sigma_{k k} \delta_{i j}\right], \\
& \sigma_{i j}=(1-d) C_{j k k}^{0} \varepsilon_{k l},
\end{aligned}
$$

where $\boldsymbol{C}_{j, k}^{0}$ is the stiffness of the undamaged material. According to this assumption, the Poisson's ratio is not affected by damage. The elastic (i.e. free) energy per unit mass of material is:

$$
\rho \psi=\frac{1}{2}(1-d) \varepsilon_{i j} C_{i j k}^{0} \varepsilon_{k l} .
$$

This energy is assumed to be the state potential. The damage energy release rate is:

$$
Y=-\rho \frac{\partial \psi}{\partial d}=\frac{1}{2} \varepsilon_{i j} C_{i j k}^{0} \varepsilon_{k l},
$$

with the rate of dissipated energy:

$$
\dot{\phi}=-\frac{\partial \rho \psi}{\partial d} \dot{d} .
$$

The second principle of thermodynamics constrains the dissipation rate to be zero or positive. In this constitutive relation (with a single damage variable), it means that damage must either increase (irreversible response) or remain constant (reversible response).

The thermodynamic framework depicted above allows also for the incorporation of damage induced anisotropy. To this end, the free energy needs to be modified in order to account for the directionality of damage. Another technique due to Fichant et al. (1997) consists in an approximation of the effective stress $\boldsymbol{\sigma}_{\mathfrak{q}}^{\boldsymbol{q}}$ :

$$
\sigma_{i j}^{t}=C_{i j k 1}^{0} \varepsilon_{u}^{e} \text { or } \sigma_{i j}^{t}=C_{i j k k}^{0}\left(C^{\text {dumaged }}\right)_{k l m n}^{-1} \sigma_{m n},
$$


where $C_{i j k l}^{0}$ is the initial stiffness of the (undamaged) material. Let us define the relation between the stress and the effective stress along a finite (or infinite) set of directions defined by unit vectors $\vec{n}$ :

$$
\begin{aligned}
\sigma & =(1-d(\vec{n})) n_{j} \sigma_{i n_{j}}^{t}, \\
\tau & =(1-D(\vec{n})) \sqrt{\sum_{i=1}^{3}\left(\sigma_{i j}^{t} n_{j}-\left(n_{k} \sigma_{k l}^{t} n_{l}\right) n_{i}\right)^{2}},
\end{aligned}
$$

where $\boldsymbol{\sigma}$ and $\boldsymbol{\tau}$ are the normal and tangential components of the stress vector respectively. Here $d(\vec{n})$ and $D(\vec{n})$ are scalar valued quantities which define the influence of damage on the relation between the effective stress and the stress vectors in direction $\vec{n}$. The overall resolved stress tensor is the solution of the virtual work equation:

$$
\begin{aligned}
& \text { find } \sigma_{i j} \text { such that } \forall \varepsilon_{i j}^{*}: \frac{4 \pi}{3} \sigma_{i j} \varepsilon_{i j}^{*}= \\
& \int_{\Omega}\left(\left[(1-d(\vec{n})) n_{k} \sigma_{k l}^{\prime} n_{l} n_{i}+(1-D(\vec{n}))\left(\sigma_{i j}^{\prime} n_{j}-n_{k} \sigma_{k l}^{\prime} n_{l} n_{i}\right)\right] \cdot \varepsilon_{i j}^{*} n_{j}\right) d \Omega,
\end{aligned}
$$

where $\boldsymbol{\Omega}$ is a sphere of radius 1 . This equation can be recast as follow:

$$
\begin{aligned}
& \text { find } \sigma_{i j} \text { such that } \forall \varepsilon_{i j}^{*}: \frac{4 \pi}{3} \sigma_{i j} \varepsilon_{i j}^{*}= \\
& \int\left(\left[(1-d(\vec{n})) E_{N} n_{k} \varepsilon_{k l}^{\prime} n_{l} n_{i}+(1-D(\vec{n})) E_{T}\left(\varepsilon_{i j}^{t} n_{j}-n_{k} \varepsilon_{k l}^{\prime} n_{l} n_{i}\right)\right] \cdot \varepsilon_{i j}^{*} n_{j}\right) d \Omega,
\end{aligned}
$$

where $E_{N}$ and $E_{T}$ are initial stiffness moduli which are functions of the Young's modulus and Poisson's ratio of the material. Eq. (11) is very similar to the equation which relates the resolved stress to the microplane stress and strains in the microplane model [Bazant and Prat 1988]. In fact, $d(\vec{n})$ and $D(\vec{n})$ can either be defined for each angular directions $\vec{n}$ independently, or can be interpolated by angular functions. In the first form, one recovers a microplane damage model and the most general form of damage induced anisotropy [Carol et al., 1991]. In the second form, damage induced anisotropy is fixed by the angular functions defining $d(\vec{n})$ and $D(\vec{n})$.It is a restriction of the first form which can be more efficient in computations as it involves less variables: a finite number of directions $\overrightarrow{\boldsymbol{n}}$ only are needed in order to define the material stiffness. It should be also noted that the integral form in Eq. (11) can be transformed again in order to arrive to a format which is very similar to the multiple fixed crack model:

$$
\sigma_{i j}=\sum_{\vec{n}} \omega^{n}\left(\left[(1-d(\vec{n})) E_{N} n_{k} \varepsilon_{k 1} n_{l} n_{i}+(1-D(\bar{n})) E_{T}\left(\varepsilon_{i j} n_{j}-n_{k} \varepsilon_{k l} n_{l} n_{i}\right)\right] \cdot n_{j}\right),
$$


where $\boldsymbol{\omega}^{n}$ is a weighting factor (see for instance the review in [de Borst, 1999]).

The evolution of $\boldsymbol{d}(\vec{n})$ and $D(\vec{n})$ for each direction $\vec{n}$ must be consistent with the second principle of thermodynamics. For isothermal conditions the rate of energy dissipation must be positive or zero. Energy dissipation is slightly more complex than for the scalar damage model (Eq. 7):

$$
\dot{\phi}=\int_{\Omega}\left[\dot{d}(\vec{n}) n_{k} \sigma_{k l}^{t} n_{l} n_{l}+\dot{D}(\vec{n})\left(\sigma_{i j}^{t} n_{j}-n_{k} \sigma_{k l}^{t} n_{l} n_{i}\right)\right] \varepsilon_{i j}^{e} n_{j} d \Omega \geq 0 .
$$

The evolution of damage is controlled by the same loading function $f$ :

$$
f(\vec{n})=\vec{n} \varepsilon^{e} \vec{n}-\varepsilon_{d}-\chi(\vec{n}),
$$

where $\boldsymbol{x}$ is a hardening - softening variable. If the damage angular functions are interpolated, e.g. with ellipsoidal functions, there are six scalar loading surfaces, one for each principal direction and for each damage variable. The hardening-softening variables are also angular functions which must be interpolated (with similar ellipsoidal functions). In the more general microplane approach, there is one loading function and one hardening-softening variable per microplane.

The loading function and the rate of the hardening-softening variable must comply finally with the Kuhn-Tucker conditions:

$$
f \leq 0, \dot{\chi} \geq 0, f \dot{\chi}=0 .
$$

\section{NONLOCAL AND GRADIENT DAMAGE}

We will first restrict attention to isotropic damage and consider a material which contains growing voids with isotropic characteristics; the nonlocal generalisation of the scalar damage model will be recalled first. Then, we will turn toward the gradient damage model and the influence of the internal length on the bifurcation condition involved in strain localisation analyses.

\subsection{Nonlocal damage model}

Consider for instance the case in which damage is a function of the positive strains (which means that it is mainly due to micro cracks opening in mode I). The following scalar called equivalent strain is introduced first (see for instance Mazars, 1984):

$$
\tilde{\varepsilon}=\sqrt{\sum_{i=1}^{3}\left(\left\langle\varepsilon_{i}\right\rangle_{+}\right)^{2}}
$$


where (\rangle$_{\%}$ is the Macauley bracket and $\boldsymbol{\varepsilon}_{\boldsymbol{i}}$ are the principal strains. In classical damage models, the loading function $f$ reads $f(\tilde{\varepsilon}, \boldsymbol{\chi})=\tilde{\varepsilon}-\chi$. The principle of nonlocal continuum models with local strains is to replace $\tilde{\boldsymbol{\varepsilon}}$ with its average:

$$
\bar{\varepsilon}(x)=\frac{1}{V_{r}(x)} \int_{\Omega} \psi(x-s) \tilde{\varepsilon}(s) d s \text { with } V_{r}(x)=\int_{\Omega} \psi(x-s) d s,
$$

where $\boldsymbol{\Omega}$ is the volume of the structure, $\boldsymbol{V}_{\boldsymbol{r}}(\boldsymbol{x})$ is the representative volume at point $x$, and $\psi(x-s)$ is the weight function, for instance:

$$
\psi(x-s)=\exp \left(-\frac{4\|x-s\|^{2}}{l_{c}^{2}}\right) .
$$

$\boldsymbol{l}_{\boldsymbol{c}}$ is the internal length of the non local continuum, $\overline{\boldsymbol{\varepsilon}}$ replaces the equivalent strain (Eq. 16) in the evolution of damage. In particular, the loading function becomes $f(\bar{\varepsilon}, \chi)=\bar{\varepsilon}-\chi$. It should be noticed that this model is easy to implement in the context of explicit, total strain models. Its implementation to plasticity and to implicit incremental relations is awkward. The local tangent stiffness operator relating incremental strains to incremental stresses becomes non symmetric, and more importantly its bandwidth can be very large due to nonlocal interactions. This is one of the reasons why gradient damage models have become popular over the past few years.

\subsection{Isotropic gradient damage model}

A simple method to transform the above nonlocal model to a gradient model is to expand the effective strain into Taylor series truncated for instance to the second order:

$$
\tilde{\varepsilon}(x+s)=\tilde{\varepsilon}(x)+\frac{\partial \tilde{\varepsilon}(x)}{\partial x} s+\frac{\partial^{2} \tilde{\varepsilon}(x)}{\partial x^{2}} \frac{s^{2}}{2 !}+\ldots . .
$$

Substitution in Eq. (17) and integration with respect to variable $s$ yields:

$$
\bar{\varepsilon}(x)=\tilde{\varepsilon}(x)+c^{2} \nabla^{2} \tilde{\varepsilon}(x) .
$$

$c$ is a parameter which depends on the type of weight function in Eq. (17). Its dimension is $L^{2}$ and it can be regarded as the square of an internal length. Substitution of the new expression of the nonlocal effective strain in the nonlocal damage model presented above yields a gradient damage model. Computationally, this model is still delicate to implement because it requires higher continuity in the interpolation of the displacement field. This difficulty can be solved with a simple trick devised by Peerlings et al. (1996): let us take the Laplacian of the right and left hand-sides of Eq. (20). 
Because the Taylor expansion in Eq. (19) was truncated to the second order, higher derivatives can be assumed to be negligible. It follows that

$$
\nabla^{2} \bar{\varepsilon}(x)=\nabla^{2} \tilde{\varepsilon}(x)+\nabla^{2}\left(c^{2} \nabla^{2} \tilde{\varepsilon}(x)\right) \approx \nabla^{2} \tilde{\varepsilon}(x) .
$$

Therefore, Eq. (20) becomes $\bar{\varepsilon}(x)-c^{2} \nabla^{2} \bar{\varepsilon}(x)=\tilde{\varepsilon}(x)$. This implicit equation which defines the nonlocal effective strain as a function of the local effective strain is easy to discretise in a finite element scheme. The implementation of the gradient damage model becomes in fact similar to the implementation of a thermomechanical (local) model in which the nonlocal effective strain replaces the nodal temperatures. This type of model has provided very good predictions of failure, for instance, of concrete structures [Peerlings et al., 1998].

Gradient damage models have also been devised differently. Frémond and coworkers (1993) started from the principle of virtual work adding the power of internal forces involving higher order terms.

A more recent approach, inspired by the mechanics of porous materials, was proposed by Pijaudier-Cabot and Burlion [1996]: assume that damage can be characterised by the variation of volume fraction of material denoted as $v(x, t)$.Failure is reached when the volume fraction is equal to zero. Starting from a reference configuration where the material is strain - free and the volume fraction is $v_{R}(x)$, the variation of the volume fraction of material is $\phi(x, t)=v(x, t)-v_{R}(x)$. This variation of volume fraction of material can be due to damage growth or straining. For constant damage, the porous material is elastic and its behaviour is modelled using the theory of elastic material with voids [Cowin and Nunziato, 1983]. The governing equations are (in the absence of body forces):

$$
\begin{aligned}
& \sigma_{i j, j}=0, \\
& h_{i, i}+g=0,
\end{aligned}
$$

where $h_{i}$ is the equilibrated stress vector, and $g$ is the equilibrated body force. These variables are related to the stresses due to centres of dilation made of three couples of opposite forces without moments acting along three mutually orthogonal directions at material points. Such forces correspond to the local pressures necessary to augment the size of an existing void, in a reversible or irreversible manner and they create a local stress distribution. These forces produce void growth and a variation of the overall volume fraction of the body. The elastic (free) energy reads:

$$
\rho \psi=\frac{1}{2} C_{i j k l} \varepsilon_{i j}^{e} \varepsilon_{u}^{e}+\beta \delta_{i j} \varepsilon_{j}^{e} \phi+\frac{1}{2} \alpha \phi_{, i} \phi_{1}+\frac{1}{2} \xi \phi^{2},
$$

where $C_{i|k|}$ are the overall stiffness coefficients of the porous material, and $(\alpha, \beta, \xi)$ are material parameters. Compared to the expression in Eq. (5), the free energy has two 
additional terms which correspond to the variation of the volume fraction of voids. The thermodynamic forces associated to the variables $\left(\boldsymbol{\varepsilon}_{\boldsymbol{y}}, \boldsymbol{\phi}, \boldsymbol{\phi}, \mathbf{)}\right)$ are defined as:

$$
\sigma_{i j}=\rho \frac{\partial \psi}{\partial \varepsilon_{i j}}, g=-\rho \frac{\partial \psi}{\partial \phi}+g_{R}, h_{i}=\rho \frac{\partial \psi}{\partial \phi_{i i}}+h_{i}^{R},
$$

where $g_{R}$ and $h_{i}^{\boldsymbol{R}}$ are the values of the equilibrated body forces and equilibrated stress vector in the reference state, respectively. Hence equilibrium in the reference (stressfree) state yields:

$$
h_{i, i}^{R}+g_{R}=0,
$$

As pointed out by Cowin and Nunziato, the stiffness coefficients and the material parameters should depend on the reference volume fraction. In order to obtain an extension of this model to the case of a damaged material, let us assume that whenever damage grows, it modifies the volume fraction of the material in the reference configuration. The variation of volume fraction is rewritten as:

$$
\begin{array}{r}
d \phi(x, t)=d \phi^{r e v}(x, t)+d \phi^{i r}(x, t), \\
\text { with }\left\{\begin{array}{l}
\phi^{r e v}(x, t)=v(x, t)-v^{R}(x, t), \\
\phi^{i r}(x, t)=v^{R}(x, t)-v^{0}(x),
\end{array}\right.
\end{array}
$$

where $\phi^{r v}$ is the reversible variation of volume fraction, $v^{R}(x, t)$ is the volume fraction of the damaged material when it is free of loads, $\phi^{\text {ir }}$ is the irreversible variation of volume fraction due to the growth of damage measured when the material is free of loads and $\boldsymbol{v}^{\mathbf{0}}$ is the initial volume fraction of the material, when damage is equal to zero. For the sake of simplicity, we assume that $\phi^{\text {ro }}$ is very small compared to $\phi^{i r}$ as damage grows. The free energy of the material is now:

$$
\rho \psi=\frac{1}{2}(1-d) C_{i j k l} \varepsilon_{i j} \varepsilon_{k l}+\psi\left(\phi^{i r}, \phi_{i}^{i r}\right) .
$$

Compared to Eq. (4), $\boldsymbol{\psi}\left(\phi^{i r}, \phi_{i, i}^{i r}\right)$ represents the variation of reversible energy due to an irreversible variation of volume fraction. In order to comply with the second principle of thermodynamics, the Clausius-Duhem inequality should be verified in all instances:

$$
\frac{d \rho \psi}{d t} \leq \sigma_{i j} \dot{\varepsilon}_{i j}+h_{i} \dot{\phi}_{, i}+g \dot{\phi} .
$$


The irreversible variation of volume fraction is measured when the body is free of loads. Therefore, we assume that $\boldsymbol{g}_{\boldsymbol{R}}$ and $\boldsymbol{h}_{\boldsymbol{i}}^{\boldsymbol{R}}$ are expressed as follow:

$$
\begin{aligned}
& h_{i}^{R}=\alpha \phi_{, i}^{i r}, \\
& g_{R}=-\xi \phi^{i r}+A .
\end{aligned}
$$

The term $A$ is the quantity which controls the irreversible variation of volume fraction of the material from the initial state $\boldsymbol{\nu}^{0}(\boldsymbol{x})$ when loads are applied. $\boldsymbol{\alpha}$ is a model parameter.

A simple (maybe simplistic) way to relate the variation of volume fraction to the decrease of stiffness of the material is to assume that $\boldsymbol{d}=-\phi^{\boldsymbol{m}}$. The relations which follow from these assumptions are derived from Eqs. $(22,24,25,29)$ :

$$
\left\{\begin{array}{l}
\sigma_{i, j}=0, \\
\alpha d d_{, i j}-\xi d=A,
\end{array} \quad \sigma_{i j}=(1-d) C_{i j k} \varepsilon_{k l} .\right.
$$

$\dot{A}$ is defined by a loading function and an evolution equation:

$$
\text { if } f(\varepsilon, \chi)=0 \text { and } \frac{\partial f(\varepsilon, \chi)}{\partial \varepsilon} \dot{\varepsilon}>0 \text { then }\left\{\begin{aligned}
\frac{\partial f(\varepsilon, \chi)}{\partial \chi} & =H \dot{\varepsilon} \\
\dot{\chi} & =-\frac{\partial f(\varepsilon, \chi)}{\partial \varepsilon} \dot{\varepsilon}
\end{aligned}\right.
$$

where $H$ is a hardening - softening modulus. In Eq. (31), $\boldsymbol{\varepsilon}$ denotes an invariant of the strain genetically. Note that $\boldsymbol{\alpha}$ in Eqs. (30) has the dimension of a length squared. It is there that an internal length has been introduced in the constitutive relations.

In the absence of body forces, the two governing equations, along with the boundary conditions, are equivalent to the following conditions: find a displacement field $\vec{u}$ and a damage field $d$ such that for any kinematically admissible virtual displacement $\vec{u} *$ and damage field $d^{*}$,

$$
\int_{\Omega} \varepsilon^{*}: \sigma d v=\int_{\alpha \Omega} \vec{u} * \vec{F} d s, \int_{\Omega}\left\{\alpha \operatorname{grad}\left(d^{*}\right) \cdot \operatorname{grad}(d)+\xi d^{*} d\right\} d v=\int_{\Omega} d^{*} \cdot A d v,
$$

where $\overrightarrow{\boldsymbol{F}}$ are external applied forces. For the finite element implementation, the displacement field components and damage field are discretised with different types of polynomials, same as in coupled thermomechanical problems. A similar variational principle can be obtained in an incremental fashion for the purpose of implementing the model within a Newton - Raphson procedure and of solving this non linear set of two coupled equations iteratively. It remains, however, that additional boundary conditions are required for solving Eqs. (32): 


$$
\left\{\begin{array}{c}
u_{i}(x, t)=\tilde{u}_{i}(x, t) \text { on } \partial \Omega_{1} \text { and } \sigma \cdot \vec{n}=\vec{\tau} \text { on } \partial \Omega_{2}, \\
\left(\partial \Omega_{1} \cup \partial \Omega_{2}=\partial \Omega \text { and } \partial \Omega_{1} \cap \partial \Omega_{2}=\varnothing\right), \\
\alpha g \operatorname{rad}(d(x, t)) \cdot \vec{n}=0 \text { on } \partial \Omega,
\end{array}\right.
$$

where $\overline{\boldsymbol{n}}$ is now the unit outward normal to the boundary $\boldsymbol{\partial \boldsymbol { \Omega }}$ of the solid $\boldsymbol{\Omega}$. The meaning of the third (natural) boundary condition remains a problem to be solved.

This gradient damage model, the one which derives from Eqs. (19-21) and the integral nonlocal model are very similar. The difference is that in the first one it is the damage variable itself on which the nonlocal treatment is applied, while in the laters, the nonlocal treatment is applied to the variable which controls damage. It follows that in the finite element implementation the damage variable is interpolated in the first model and that the nonlocal effective strain is interpolated in the second.

\subsection{Strain localisation analysis}

In order to exhibit the regularisation properties of the gradient damage model, we will investigate the occurrence of bifurcation in an infinite body from a homogeneous state of deformation and damage, denoted as $\left(\varepsilon_{i j}^{0}, d^{0}\right)$. Monotonic loading is assumed and body forces are omitted. Apart from the trivial solution where the strain and damage remain homogeneous, we look for velocities $\left(\dot{u}_{1}, \dot{u}_{2}, \dot{u}_{3}\right)$ and a damage rate distribution $\dot{d}$ of the form:

$$
\dot{u}_{i}=U_{i} \exp \left(J \omega\left(n_{l} x_{l}\right)\right), \dot{d}=D \exp \left(J \omega\left(n_{l} x_{l}\right)\right),
$$

where $U_{i}(\mathrm{i}=1,2,3)$ and $D$ are unknown constants, $\boldsymbol{\omega}$ is an unknown angular frequency, $\vec{n}$ is an unknown normal vector, and $J$ is the imaginary constant such that $J^{2}=-1$. Substitution of Eqs $(30,34)$ in the governing equations of equilibrium (Eqs. 33) yields the following homogeneous system of equations:

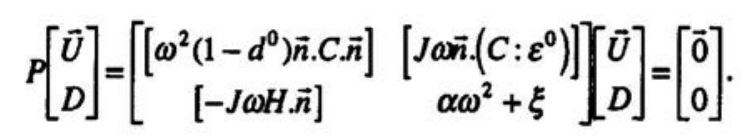

$P$ is a $4 \times 4$ matrix. This system admits non trivial solution if $\operatorname{det}(P)=0$, which is the bifurcation condition. The bifurcation condition should yield a normal vector $\overrightarrow{\boldsymbol{n}}$ for each value of the angular frequency. One can check easily that if $\alpha=0$, the solution is independent of the angular frequency which can be fixed arbitrarily. The square root of $\boldsymbol{\alpha}$ is an internal length of the continuum which selects the wave length of the localised solution and scales the size of the localisation band. It plays exactly the same role as the internal length in other localisation limiters. 


\section{CLOSURE : EXTENSION TO ANISOTROPIC GRADIENT DAMAGE}

In the anisotropic damage models devised in the first section of this contribution, the evolution of damage is directional. For instance one loading function $f(\vec{n})$ is considered for each direction $\overline{\boldsymbol{n}}$ in Eq. (14). Combining Eq. (14) and the definition of the nonlocal strain in Eq. (19-21) can be done: for each direction $\overrightarrow{\boldsymbol{n}}$, Eq. (14) can replaced by:

$$
f(\vec{n})=\bar{\varepsilon}_{N}-\chi(\vec{n}), \quad \bar{\varepsilon}_{N}-c^{2} \nabla^{2}\left(\bar{\varepsilon}_{N}\right)=\vec{n} \cdot \varepsilon^{e} \cdot \vec{n} .
$$

For each direction in which damage growth is defined, a nonlocal variable is introduced. This extension is straightforward in the context of microplane models where directions $\vec{n}$ are defined arbitrarily and fixed in the analysis. There are as many nonlocal variables as directions considered and these variables are interpolated throughout the finite element mesh, same as for the isotropic gradient damage model. The usual set of equilibrium equations is complemented with $p \times m$ equations where $p$ is the number of directions and $m$ is the number of damage variable per direction [Kuhl et al., 1998].

For the orthotropic damage model proposed by Fichant et al., three directions are considered only at each material point. The directional distribution of damage is reconstructed by interpolation in between those principal directions. Hence, there is the complexity that the directions change from one finite element to another and might rotate in the course of loading too. A similar problem is encountered in nonlocal rotating crack models [de Borst, 1999]. Conceptually, this feature does not change anything in the discretisation, except that one has to keep track of the orientation of principal directions of damage. This is in fact the price to pay for the reduction of equations (and degrees of freedom) to be solved.

\section{BIBLIOGRAPHY}

[Bazant, 1976] Bazant, Z.P., Instability, ductility and size effect in strain softening concrete, J. Engng. Mech. ASCE, Vol. 102, pp. 331-344.

[Bazant and Prat, 1988] Bazant, Z.P., and Prat, P.C., Microplane model for brittleplastic material I: theory, J. Engng. Mech. ASCE, Vol. 114, pp. 1672-1702.

[de Borst and Muhlhaus, 1992] de Borst, R., and Muhlhaus, H.B., Gradient dependent plasticity : formulation and algorithmic aspects, Int. J. Num. Meth. in Engrg., Vol. 35, pp. 521-539.

[de Borst, 1991] de Borst, R., Simulation of strain localisation: a reappraisal of the cosserat continuum, Eng. Comput., Vol. 8, pp. 317-332.

[de Borst, 1999] de Borst, R., Isotropic and anisotropic damage models for concrete fracture, in Mechanics of Quasi-Brittle Matrials and Structures, G. Pijaudier-Cabot et al. Eds., Hermes Sci. Pubs., pp. 39-56.

[Carol et al., 1991] Carol, I., Bazant, Z.P., and Prat, P.C., Geometrical damage tensor based on microplane model, J. of Engrg. Mech. ASCE, Vol. 117, pp. 2429-2448. 
[Cowin and Nunziato, 1983] Cowin, S.C., and Nunziato, J.W., Linear elastic materials with voids, J. of Elast., Vol. 13, pp. 125-147.

[Feenstra, 1993] Feenstra, P.H., Computational Aspects of Biaxial Stress in Plain and Reinforced Concrete, Dissertation, Delft University of Technology, the Netherlands.

[Fichant et al., 1997] Fichant, S., Pijaudier-Cabot, G., La Borderie, C., Continuum damage modelling: approximation of crack induced anisotropy, Mech. Res. Comm., Vol. 24, pp. 109-114.

[Frémond and Nedjar, 1993] Frémond, M., Nedjar, B., Endommagement et principe des puissances virtuelles. Cornptes Rendus Acad. Sci., série II, pp. 857-864.

[Joseph et al. 1985] Joseph, D., Renardy, M., and Saut, J.C., Hyperbolicity and change of type in the flow of viscoelastic fluids, Arch, for Rational Mech. and Analysis, Vol. 87, pp. 213-251.

[Kuhl et al. 1998] Kuhl, E., Ramm, E., and de Borst, R., Anisotropic damage model with the microplane model, in Computational Modelling of Concrete Structures, de Borst et al. Eds., Balkema Pubs, pp. 103-112.

[Lemaitre, 1992] Lemaitre J., A Course on Damage Mechanics, Springer Verlag.

[Needleman, 1988] Needleman, A., material rate dependence and mesh sensitivity in localization problems, Comp. Meth. Appl. Mech. Engrg., Vol. 67, pp. 69-86.

[Mazars, 1984] Mazars, J., Application de la mécanique de l'endommagement au comportement non lineaire et à la rupture du béton de structure, Thèse de Doctoral ès Sciences, Université Paris 6.

[Peerlings et al., 1996] Peerlings, R.H., de Borst, R., Brekelmans, W.A.M., de Vree, J.H.P., Gradient enhanced damage for quasi-brittle materials, Int. J. Num. Meth. Engrg., Vol. 39, pp. 3391-3403.

[Peerlings et al., 1998] Peerlings, R.H., de Borst, R., Brekelmans, W.A.M, Geers, M.G.D., Gradient enhanced modelling of concrete fracture, Mech. Coh.-Frict. Mat., Vol. 3, pp. 323-343.

[Pijaudier-Cabot and Bazant, 1987] Pijaudier-Cabot, G., and Bazant, Z.P., Nonlocal damage theory, J. of Engrg. Mech. ASCE, Vol. 113, pp. 1512 -1533.

[Pijaudier-Cabot and Benallal, 1993] Pijaudier-Cabot, G., and Benallal, A., Strain localisation and bifurcation in a nonlocal continuum, Int. J. Solids Struct., Vol. 30, pp. 1761-1775.

[Pijaudier-Cabot and Burlion, 1996] Pijaudier-Cabot, G., and Burlion, N., Damage and localisation in elastic materials with voids, Int. J. Mech. ofCohes.-Frict. Mat., Vol.1, pp. 129-144.

[Rice, 1976] Rice, J.R., The localisation of plastic deformation, in Proc. Int. Congress of Theor. andAppl. Mech., Delft, The Netherlands, Vol. 1, pp. 207-220.

[Sluys, 1992] Sluys, L.J., Wave Propagation, Localisation and Dispersion in Softening Solids, Doctoral Dissertation, Delft University of Technology, The Netherlands. 